\title{
Obesity status and lifestyle pattern of pre and post menopausal urban-employed women of Mysore city, India
}

\begin{abstract}
Menopause doubles the risk of many traditional CVD risk factors, including changes in body fat distribution from gynoid to android pattern, atherogenic lipid profile, hypertension and diabetes. Given the diversified roles/ responsibilities of women, employment would result in a net increase in her work timings with adverse effects on health and nutritional status. Hence, the objective of the study was to assess the obesity status, nutritional status and health practices of urban-employed pre and postmenopausal women from various professions belonging to Mysore city, Karnataka state, India. A comparative cross sectional study was carried out between pre and postmenopausal women employed in educational institutions, health care sectors, and government offices, situated in Mysore city. A total of 443 pre and post-menopausal employed women who volunteered to participate were administered a set of pre- tested questionnaire, to elicit information on demography, socio economic status, personal history, health practices and family history for cardiovascular disease. Descriptive statistical analysis was carried out in the present study. Irrespective of menopausal status, profession or age majority of the subjects had higher BMI, WHR, WC, low protein status as indicated by low MUAC and MUAMC and high body fat status a predisposition factor for CVD. BMI and WHR were significantly higher $(\mathrm{p}<0.001)$ in post menopausal women as compared to pre menopausal women, also, Post $\mathrm{M}$ women from teaching profession had significantly higher BMI as compared to other professional groups. The dietary preferences for snacking and higher intake of coffee and tea that increased the consumption of sugar and salt per day, low physical activity and family history for cardiovascular events and risk factors can contribute to acceleration of altered cardio bio markers and thereby promote adverse health effects. Lack of motivation and awareness to undergo annual cardiac evaluation, places them at a higher risk for cardiac events in the future. The study indicates that hereditary factors may be super imposed by environmental factors for development of risk factors for cardiovascular disease, and needs early detection in the form of annual medical check - up and self-assessment of body measures for all women above $30 \mathrm{y}$ of age.
\end{abstract}

Keywords: menopausal women, cardiovascular disease, dietary assessment, obesity, diabetes, chronic diseases, lifestyle disorders
Volume 6 Issue 2 - 2017

\author{
Charlotte G Karunakaran, Asna Urooj \\ Department of Studies in Food Science Nutrition, University of \\ Mysore, India
}

Correspondence: Asna Urooj, Department of Studies in Food Science Nutrition, University of Mysore, Manasagangothri, Mysore 570006, India, Tel 918212419632,

Email asnaurooj@foodsci.uni-mysore.ac.in

Received: November II, 2016 | Published: February 02, 2017
Abbreviations: CVD, cardio vascular diseases; BMI, body mass index; WHR, waist hip ratio; SES, socio-economic status; WC, waist circumference; TSF, tri fold thickness; MUAC, mid upper arm circumference; MUAMC, mid upper arm muscle circumference; $\mathrm{NNMB}$, national nutrition monitoring bureau; $\mathrm{CHD}$, coronary heart disease

\section{Introduction}

The health needs of the world's population are undergoing dramatic changes. Many developing countries of the world are undergoing an 'epidemiological transition' with regard to non-communicable diseases especially obesity. The prevalence of obesity and metabolic syndrome is rapidly increasing in India and other South Asian countries, leading to increased mortality and morbidity due to CVD and T2DM., ${ }^{1,2} \mathrm{~A}$ study on prevalence of overweight and in children and adults during 1980-2013, projects that India is just behind US and China in this global hazard list of top 10 countries with highest number of obese people ${ }^{3}$ Changes in food and nutrient intakes are associated with rapid industrialization and urbanization of populations in developing countries. Lifestyle factors such as reduced physical activities, stress, smoking and excessive consumption of alcohol underlie the emerging epidemics of a range of chronic diseases such as obesity, coronary heart disease, hypertension, stroke, non-insulin dependent diabetes, obesity etc. Escalations of nutrition related chronic diseases are considered to be an urban phenomenon. The largely implicated urban phenomenons are changes in dietary patterns, occupational patterns, lifestyle changes and possibly the impact of environmental factors. ${ }^{4-6}$

Menopause is the strongest biological transitory phase in a women's life. The physiological and psychological changes in menopause also influence the food intake and nutritional status of women. Menopause compounds many traditional CVD (Cardio vascular Diseases) risk factors, including changes in body fat distribution from gynoid to android pattern, atherogenic lipid profile, hypertension and diabetes. ${ }^{7,8}$

Changing dimensions in socioeconomic status has enhanced opportunities for employment of women in India. Given the diversified roles/ responsibilities of women, employment would result in a net increase in her work timings with adverse effects on health and nutritional status. Studies on the health effects on women's employment have always generated great interest. 


\section{Objective}

To assess the obesity status as influenced by menopausal status, profession and age in an urban-employed pre and post menopausal women belonging to Mysore city. The lifestyle pattern of the subjects was also studied.

\section{Methodology}

\section{Sampling design and survey methods}

A cross sectional study was carried out on women employed in educational institutions, health care sectors, and government offices, situated in Mysore city. A total of 443 pre and post-menopausal employed women who volunteered to participate were administered a set of pre-tested questionnaire, to elicit information on demography, socio economic status, personal history, health practices and family history for CVD. An assessment on the somatic status viz; height $(\mathrm{cm})$, weight $(\mathrm{kg})$, waist circumference $(\mathrm{cm})$, hip circumference $(\mathrm{cm})$ was carried out on the subjects and relevant indices was calculated BMI (body mass index) and WHR (waist hip ratio). Selection of the subjects, recruited for the study from various institutions is presented in Table 1.

Table I Selection of study group

\begin{tabular}{lll}
\hline Institution & No. of subjects & No. of institutions \\
\hline Educational & 181 & $9\{$ Govt -2 , Private -7$\}$ \\
Hospitals & 122 & $4\{$ Govt -2 , Private -2$\}$ \\
Telecom sector & 120 & $2\{$ Govt $\}$ \\
Offices & 20 & $2\{$ Govt $\}$ \\
Total & 443 & 17 \\
\hline
\end{tabular}

\section{Screening and selection of subjects}

The study objective was informed to the employees by way of pamphlets distributed at work places, as well as through introductory group lectures. Individuals willing to participate and belonging to the age group between 20-60years, with pre and post menopausal history were included as subjects.

\section{Inclusion and exclusion criteria}

The Pre-menopausal women (Pre $\mathrm{M}$ ) were required to be in menstruating state, ( $>9$ menstrual cycles/ year), non pregnant and without a history of any previous cardiac event or stroke.

The Post menopausal women (Post-M) were required to have complete cessation of menstrual cycles (eumenorhiac >1year), not on any hormonal replacement therapy, and without history of any previous cardiac event or stroke.

\section{Data collection}

A questionnaire was formulated to elicit information on the following aspects

a. Demography-Age, Religion, Marital status, Occupation, Education.

b. Socio-economic status (SES)-Number of earning members, Economic status.

c. Personal history-Pre/Post menopausal history. d. Anthropometric measures - Height $(\mathrm{cm})$, Weight $(\mathrm{kg})$ waist and hip circumference $(\mathrm{cm})$, Mid arm circumference $(\mathrm{cm})$ and Skin fold (mm) measurements.

e. Health practices - Dietary habits, Physical activity, sleep schedule, and medical history.

f. Family history of cardiovascular diseases.

\section{Anthropometric measurements}

The measurements and their indices were recorded and calculated according to standard procedures., ${ }^{9,10}$

a. Height (cm): was measured with the subject standing, back to a (stadio meter) in the base feet. Feet were kept parallel with the heels together. The moving arm of the stadio meter was lowered to touch the top of the head and height was measured to the nearest $1.0 \mathrm{~mm}$.

b. Weight (kg): was measured to the nearest $0.005 \mathrm{~kg}$ with a weighing machine, which was calibrated daily by using known $5 \mathrm{~kg}$ weights.

c. MUAC (cm): was measured on the right arm at the point between the tips of olecranon, elbow bent at 90 degree.

d. Skin fold thickness $(\mathbf{m m})$ : was measured according to the protocol using skin fold callipers (beta technology incorporated; USA)

e. Triceps Skinfold $(\mathbf{m m})$ : was measured at mid-point of right arm elbow, bent at 90 degree on the lateral side.

f. Waist circumference $(\mathbf{c m})$ : was measured midway between the lateral ribs and iliac crests. The subjects were asked not to tuck their stomach in, and the measurement was taken in gentle expiration. Their clothes were loosened around the waist area.

g. Hip Circumference (cm): was measured at the widest part over the trochanters with the Feet kept $25-30 \mathrm{~cm}$ apart.

\section{Anthropometric data was used for the calculation of the following}

Body mass index $(\mathrm{BMI})=$ weight $(\mathrm{Kg}) /$ height $(\mathrm{Mts})^{2}$

a. Mid upper arm muscle circumference $($ MUAMC $)=$ MUAC $\mathrm{TSF} \times 3.14^{10}$

Where TSF - Triceps skin fold,

MUAC - mid upper arm circumference $(\mathrm{cm})$

b. Waist Hip Ratio $($ WHR $)=$ waist $(\mathrm{cm})$ hip $(\mathrm{cm})$.

\section{Statistical analysis}

Descriptive statistical analysis was carried out in the present study. Results on continuous measurements are presented as Mean $\pm \mathrm{SD}$ (Min-Max) and results on categorical measurements is presented in Number (\%). Significance is assessed at $5 \%$ level of significance. Analysis of variance (ANOVA) has been used to find the significance of study parameters between three or more groups of subjects, Chisquare/ Fisher Exact test has been used to find the significance of study parameters on categorical scale between three groups. Student $t$ test (two tailed, independent) has been used to find the significance of investigation parameter between two groups of subjects. 


\section{Results}

The base line data of the subjects is depicted in Figure 1. The subjects were all literates with varied levels of basic and professional degrees. $75.7 \%$ of the teachers were post graduates. $57.4 \%$ of health care professional were diploma holders and belonged to nursing profession, remaining $43.6 \%$ were qualified graduates/ post gradates medical doctors and paramedical professionals. $92.1 \%$ of subjects from clerical category had only 10-12 y of basic education, with additional diploma pertaining to their job requirement

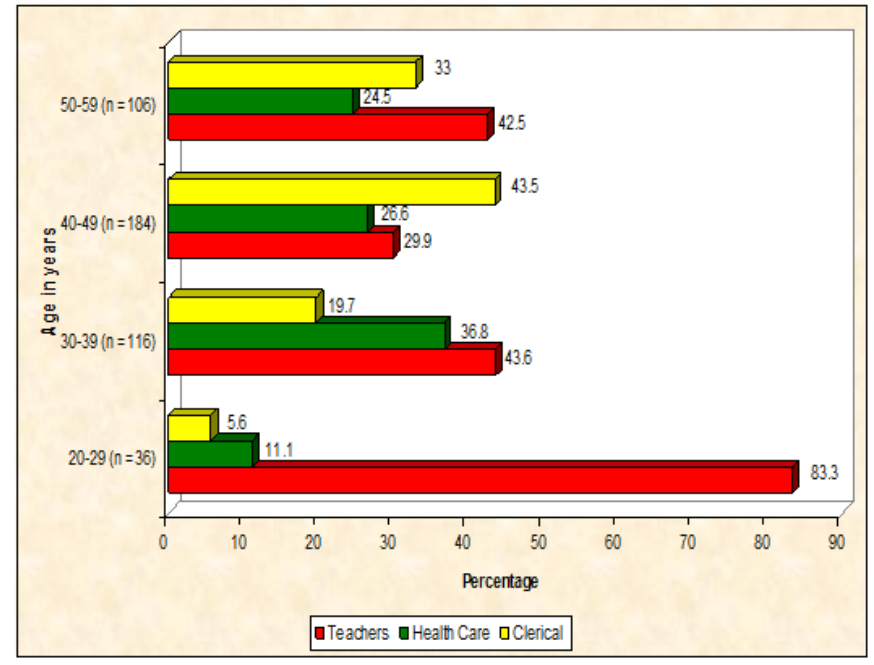

Figure I Distribution of study group: Menopausal status and Profession.

\section{Somatic status}

Anthropometric parameters, viz; Weight, Height, Waist circumference (WC), TSF, MUAC and their indices - BMI, WHR and MUAMC of the study group by their profession and menopausal status are presented in Tables $2 \& 3$. Somatic status on the basis of menopausal status within each professional group showed that Post $\mathrm{M}$ women in all the professional groups had higher measurements of WC, Hip circumference, BMI, WHR, TSF, MUAC and MUAMC as compared to the Pre M group of women. However, MUAC and MUAMC were lower than the standard values in both Pre M and Post $\mathrm{M}$ groups while, TSF was higher than the standard values in both groups.

Mean weight (Table 4) showed a gradual increase with increase in age. Indicators of overweight and obesity showed an increasing trend with increase in age. Cardiovascular disease risk parameters viz; BMI, WHR and WC also showed an increasing trend with increase in age. However, fat status as measured by TSF was found to be high in all age groups and body protein status as measured by MUAC \& MUAMC was observed to be below normal in all the age groups. It was of interest to note that according to standards for Asians viz; (BMI $>23$, waist circumference $>80 \mathrm{~cm}$,) all women more than $30 \mathrm{y}$ of age had higher mean BMI and WC (25.3 \pm 3.9 and $80.3 \pm 15.5)$.

\section{Assessment of general obesity}

Classification of BMI according to WHO 1998 guidelines, indicated that younger age group women (20-29 y) (Table 5) were significantly underweight $(\mathrm{p}=0.005)$ and older women $(>40 \mathrm{y})$ were significantly overweight $(\mathrm{p}=0.010)$ and or obese. $(\mathrm{p}=0.002)$. Classification according to standards proposed for Asian Indians showed significant $(\mathrm{p}=0.008)$ number of underweight women belonged to younger age group i.e., 20-29y. Those at risk for obesity were seen in all age groups. Grade I obesity was significantly higher $(\mathrm{p}=0.010)$ in the 40 49 age group. While, grade II obesity was significantly high $(\mathrm{p}=0.001)$ in women aged $>50 \mathrm{y}$.

Table 2 Statistical Significance of anthropometric parameters between Pre $M$ and Post $M$ within the profession

\begin{tabular}{llll}
\hline \multirow{2}{*}{ Anthropometric Parameters } & \multicolumn{3}{c}{ Significance between pre and post menopause } \\
\cline { 2 - 4 } & Teachers & Health Care & Clerical \\
\hline Age $($ years $)$ & $<0.00 I^{* *}$ & $<0.00 I^{* *}$ & $<0.00 I^{* *}$ \\
Height $(\mathrm{cm})$ & $0.057+$ & 0.29 & 0.164 \\
Weight $(\mathrm{kg})$ & 0.25 I & $0.055+$ & $0.032^{*}$ \\
BMI $\left(\mathrm{kg} / \mathrm{m}^{2}\right)$ & $0.026^{*}$ & $0.010^{* *}$ & $0.003^{* *}$ \\
Waist Circ $(\mathrm{cm})$ & $0.014^{*}$ & 0.145 & $0.005^{* *}$ \\
HIP $(\mathrm{cm})$ & 0.325 & 0.187 & $0.028^{*}$ \\
WHR & $0.003^{* *}$ & 0.426 & 0.127 \\
MUAC $(\mathrm{cm})$ & 0.572 & 0.569 & $0.053+$ \\
TSF $(\mathrm{mm})$ & 0.251 & 0.375 & 0.697 \\
MUAMC $(\mathrm{cm})$ & 0.193 & 0.854 & 0.255 \\
\hline
\end{tabular}

values are obtained using the student $\mathrm{t}$ test (two tailed, independent)

*Moderately significant $0.01<\mathrm{P} \leq 0.05$

**Highly significant $\mathrm{P} \leq 0.01$

+Suggestive significance 
Table 3 SD \pm Mean Anthropometric Measurements: Pre M vs Post M

\begin{tabular}{llllll}
\hline \multirow{2}{*}{ Anthropometric parameters } & \multicolumn{2}{c}{ Pre-menopause } & \multicolumn{2}{c}{ Post-menopause } & \multirow{2}{*}{ P value } \\
\cline { 2 - 5 } & Mean & SD & Mean & SD & \\
\hline Height $(\mathrm{cm})$ & 156.7 & 6.4 & 155.1 & 7 & $0.016^{*}$ \\
Weight $(\mathrm{kg})$ & 59.6 & 9.5 & 63 & 9.2 & $0.00 \mathrm{I}^{* *}$ \\
BMI $\left(\mathrm{kg} / \mathrm{m}^{2}\right)$ & 24.3 & 3.7 & 26.3 & 4 & $<0.00 \mathrm{I} * *$ \\
Waist Circumference $(\mathrm{cm})$ & 78.7 & 14.6 & 84.4 & $1 \mathrm{I} . \mathrm{I}$ & $<0.00 \mathrm{I} * *$ \\
HIP $(\mathrm{cm})$ & 96.7 & 13.8 & 100 & 8.8 & $0.012^{*}$ \\
WHR & 0.8 & 0.1 & 0.8 & 0.1 & $0.002^{* *}$ \\
MUAC $(\mathrm{cm})$ & 26.8 & 3.3 & 27.3 & 3.3 & 0.149 \\
TSF $(\mathrm{mm})$ & 24.4 & 6.2 & 24.5 & 6.1 & 0.812 \\
MUAMC $(\mathrm{cm})$ & 19.1 & 3 & 19.5 & 3.3 & 0.303 \\
\hline
\end{tabular}

$P$ values are obtained using the student $t$ test (two tailed, independent)

*Moderately significant $0.01<p \leq 0.05$

**Highly significant $\mathrm{p} \leq 0.01$

Table 4 SD \pm Mean Anthropometric measurements of the subjects according to age

\begin{tabular}{|c|c|c|c|c|}
\hline Parameter & $20-29 n=36$ & $30-39 n=117$ & $40-49 n=184$ & $50-59 n=106$ \\
\hline Age (yrs) & $26.3 \pm 1.6$ & $34.9 \pm 2.8$ & $44.2 \pm 2.8$ & $54.5 \pm 2.8$ \\
\hline Height $(\mathrm{cm})$ & $159.2 \pm 5.6$ & $155.7 \pm 7.0$ & $\mid 56.0 \pm 6.1$ & $154.9 \pm 7.0$ \\
\hline Weight(kg) & $55.2 \pm 11.0$ & $58.9 \pm 9.4$ & $61.4 \pm 9.2$ & $62.3 \pm 9.1$ \\
\hline BMI & $21.7 \pm 3.8$ & $23.9 \pm 3.4$ & $25.3 \pm 3.9$ & $26.0 \pm 3.9$ \\
\hline Waist(cm) & $71.4 \pm 15.7$ & $79.0 \pm 11.4$ & $80.3 \pm 15.5$ & $84.2 \pm 11.2$ \\
\hline $\mathrm{Hip}(\mathrm{cm})$ & $91.9 \pm 14.0$ & $96.9 \pm 11.3$ & $97.5 \pm 14.8$ & $99.9 \pm 8.4$ \\
\hline WHR & $0.77 \pm 0.1$ & $0.81 \pm 0.1$ & $0.82 \pm 0.1$ & $0.84 \pm 0.1$ \\
\hline $\mathrm{TSF}(\mathrm{mm})$ & $23.3 \pm 5.8$ & $24.3 \pm 6.2$ & $25.0 \pm 6.4$ & $23.8 \pm 5.9$ \\
\hline $\operatorname{MUAC}(\mathrm{cm})$ & $21.5 \pm 3.1$ & $26.7 \pm 3.2$ & $26.9 \pm 3.4$ & $27.3 \pm 3.0$ \\
\hline $\operatorname{MUAMC}(\mathrm{cm})$ & $18.2 \pm 2.2$ & $19.2 \pm 2.7$ & $19.1 \pm 3.4$ & $19.7 \pm 3.2$ \\
\hline
\end{tabular}

TSF: 16.5

MUAC: 28.5

MUAMC: 23.2

Table $\mathbf{5}$ Obesity as measured by BMI with age distribution

\begin{tabular}{|c|c|c|c|c|c|c|}
\hline \multirow{2}{*}{ BMI $\left(\mathrm{kg} / \mathrm{m}^{2}\right)$ range } & \multicolumn{4}{|c|}{ Age in years } & \multirow{2}{*}{ Total } & \multirow{2}{*}{ PValue } \\
\hline & $20-29$ & $30-39$ & $40-49$ & $50-59$ & & \\
\hline \multicolumn{7}{|l|}{ WHO guidelines ( 1998 ) } \\
\hline < 8.5 (Under weight) & $6(16.7)$ & $6(5.1)$ & $8(4.3)$ & $2(1.9)$ & $22(4.9)$ & $0.005 * *$ \\
\hline I8.5-24.9 (Normal) & $23(63.9)$ & $6 I(52.1)$ & $75(40.8)$ & $37(34.9)$ & 196(44.2) & $0.004 * *$ \\
\hline 25.0-29.9 (Over weight) & $6(16.7)$ & $46(39.3)$ & $84(45.6)$ & $48(45.3)$ & $\mid 84(4 \mid .5)$ & $0.010^{*}$ \\
\hline 30.0-30.9 (Obesity) & $\mathrm{I}(2.8)$ & $4(3.4)$ & $17(9.2)$ & $18(16.9)$ & $40(9.0)$ & $0.002^{* *}$ \\
\hline 240(Extreme Obesity) & 0 & 0 & 0 & $\mathrm{I}(0.9)$ & $I(0.3)$ & - \\
\hline
\end{tabular}


Tale Continued....

\begin{tabular}{|c|c|c|c|c|c|c|}
\hline \multirow{2}{*}{ BMI $\left(\mathrm{kg} / \mathrm{m}^{2}\right)$ range } & \multicolumn{4}{|c|}{ Age in years } & \multirow{2}{*}{ Total } & \multirow{2}{*}{ P Value } \\
\hline & $20-29$ & $30-39$ & $40-49$ & $50-59$ & & \\
\hline \multicolumn{7}{|c|}{ Asia pacific standard (2000) } \\
\hline < 8.5 (Underweight) & $6(16.7)$ & $6(5.1)$ & $8(4.3)$ & $2(1.9)$ & $22(4.9)$ & $0.005^{* *}$ \\
\hline 18.5-22.9 (Normal) & $18(50.0)$ & $3 I(26.5)$ & $38(20.6)$ & $21(19.8)$ & $108(24.4)$ & $0.008 * *$ \\
\hline 23.0-24.9 (Overweight) & $5(13.9)$ & $30(25.6)$ & $37(20.1)$ & $16(15.1)$ & $88(19.8)$ & 0.188 \\
\hline 25.0-29.9 (Obesity I) & $6(16.7)$ & $46(39.3)$ & $84(45.7)$ & $48(45.3)$ & $184(4 \mid .5)$ & $0.010^{*}$ \\
\hline$\geq 30$ (Obesity II) & $\mathrm{I}(2.8)$ & $4(3.4)$ & $17(9.2)$ & $19(17.9)$ & $4 I(9.2)$ & $0.00 I^{* *}$ \\
\hline Total & $36(100.0)$ & $117(100.0)$ & $184(100.0)$ & $106(100.0)$ & $443(100.0)$ & - \\
\hline
\end{tabular}

Results are presented as No (\%)

$P$ values are obtained using the Chi-square test/2x4 Fisher Exact

*Moderately significant $0.01<P \leq 0.05$

**Strongly significant $\mathrm{P} \leq 0.01$

Similarly classifying the women according to Menopausal status (Table 6) showed that significantly higher number of Pre M women

Table 6 Comparison of BMI according to WHO and Asia Pacific guidelines had normal BMI and significantly more number of Post $\mathrm{M}$ women ( $\mathrm{p}=$ $<0.001$ ) were obese.

\begin{tabular}{|c|c|c|c|c|}
\hline \multirow{2}{*}{ BMI $\left(\mathrm{kg} / \mathrm{m}^{2}\right)$ range } & \multicolumn{2}{|c|}{ Menopausal status } & \multirow{2}{*}{ Total } & \multirow{2}{*}{ P Value } \\
\hline & Pre $M$ & Post M & & \\
\hline \multicolumn{5}{|c|}{ WHO Guidelines (1998) } \\
\hline < 8.5 (Under weight) & 19(5.9) & $3(2.4)$ & $22(4.9)$ & 0.124 \\
\hline I8.5-24.9 (Normal) & I58(49.5) & $38(30.6)$ & $196(44.2)$ & $<0.001 * *$ \\
\hline 25.0-29.9 (Over weight) & $124(38.9)$ & $60(48.4)$ & $\mid 84(4 \mid .5)$ & $0.068+$ \\
\hline 30.0-30.9 (Obesity) & $18(5.6)$ & $22(17.7)$ & $40(9.0)$ & $<0.001 * *$ \\
\hline$\geq 40$ (Extreme Obesity) & 0 & $\mathrm{I}(0.8)$ & $I(0.3)$ & 0.28 \\
\hline \multicolumn{5}{|c|}{ Asia pacific standard (2000) } \\
\hline <18.5 (Underweight) & 19(5.9) & $3(2.4)$ & $22(4.9)$ & 0.124 \\
\hline 18.5-22.9 (Normal) & $86(26.9)$ & $22(17.7)$ & $108(24.4)$ & $0.043 *$ \\
\hline 23.0-24.9 (Over weight) & $72(22.6)$ & $16(12.9)$ & $88(19.8)$ & $0.022 *$ \\
\hline 25.0-29.9 (Obesity I) & $124(38.9)$ & $60(48.4)$ & $184(4 \mid .5)$ & $0.068+$ \\
\hline Z30 (Obesity II) & $18(5.6)$ & $23(\mid 8.5)$ & $4 I(9.2)$ & $<0.00 I^{* *}$ \\
\hline Total & $319(100.0)$ & $124(100.0)$ & $443(100.0)$ & - \\
\hline
\end{tabular}

Results are presented as No (\%)

$P$ values are obtained using the Chi-square test/ $2 \times 2$ Fisher Exact test

*Moderately significant $0.01<\mathrm{P} \leq 0.05$

**Strongly significant $\mathrm{P} \leq 0.01$

+Suggestive significance

\section{Assessment of central obesity}

The general measure of central obesity is by measuring the waist and hip in centimetres and arriving at waist hip ratio (WHR). Central obesity can result in hyperinsulinaemia causing more chances of diabetes mellitus and coronary heart disease. It is not only common,

but also more dangerous for Asian Indians. Hence, 0.80 for women and 0.90 for men has been accepted as the cut off for WHR. Data on waist circumference (WC) and waist hip ratio (WHR), established risk factors for $\mathrm{CAD}$, according to age and menopausal status is presented in Tables $7 \& 8$. 
Table 7 Abdominal obesity as measured by WHR by age

\begin{tabular}{|c|c|c|c|c|c|c|}
\hline \multirow{2}{*}{ Range } & \multicolumn{4}{|c|}{ Age in years } & \multirow{2}{*}{ Total } & \multirow{2}{*}{$P$ value } \\
\hline & $20-29$ & $30-39$ & $40-49$ & $50-59$ & & \\
\hline \multicolumn{7}{|c|}{ Waist hip ratio } \\
\hline$\leq 0.80$ & $25(69.4)$ & $53(45.3)$ & $67(36.4)$ & $34(32.1)$ & I79(40.4) & $<0.001 * *$ \\
\hline $0.80-0.85$ & $6(16.7)$ & $39(33.3)$ & $67(36.4)$ & $26(24.5)$ & $138(3 \mid .2)$ & $0.040 *$ \\
\hline $0.85-0.90$ & $2(5.6)$ & $16(13.7)$ & $30(16.3)$ & $20(18.9)$ & $68(15.3)$ & $<0.001 * *$ \\
\hline$>0.90$ & $3(8.3)$ & $9(7.7)$ & $20(10.9)$ & $26(24.5)$ & $58(13.1)$ & $0.00 I^{* *}$ \\
\hline \multicolumn{7}{|c|}{ Waist (cm) } \\
\hline$<80.0$ & $26(72.2)$ & $61(52.1)$ & $80(43.5)$ & $35(33.0)$ & $202(45.6)$ & $<0.001 * *$ \\
\hline $80-85$ & $4(11.1)$ & $31(26.5)$ & $40(21.7)$ & $20(18.9)$ & $95(21.4)$ & 0.214 \\
\hline $85-88$ & $2(5.6)$ & $9(7.7)$ & $17(9.2)$ & $13(12.3)$ & $4 I(9.3)$ & 0.558 \\
\hline$>88$ & $4(11.1)$ & $16(13.7)$ & $47(25.5)$ & $38(35.8)$ & $105(23.7)$ & $<0.001 * *$ \\
\hline Total & $36(100.0)$ & $117(100.0)$ & $184(100.0)$ & $106(100.0)$ & $443(100.0)$ & - \\
\hline
\end{tabular}

Results are presented as No (\%)

$P$ values are obtained using the Chi-square test/ $2 \times 4$ Fisher Exact test

*Moderately significant $0.01<\mathrm{P} \leq 0.05$

$* *$ Strongly significant $\mathrm{P} \leq 0.0 \mathrm{I}$

Table 8 WHR values based on menopausal status

\begin{tabular}{|c|c|c|c|c|}
\hline \multirow{2}{*}{ Range } & \multicolumn{2}{|c|}{ Menopausal status } & \multirow{2}{*}{ Total } & \multirow{2}{*}{ P Value } \\
\hline & Pre M & Post M & & \\
\hline \multicolumn{5}{|c|}{ Waist Hip Ratio } \\
\hline$\leq 0.80$ & $|4|(44.2)$ & $38(30.6)$ & $179(40.4)$ & $0.009 * *$ \\
\hline $0.80-0.85$ & $105(32.9)$ & $33(26.6)$ & $138(31.2)$ & 0.198 \\
\hline $0.85-0.90$ & $44(13.8)$ & $24(19.4)$ & $88(19.9)$ & 0.145 \\
\hline$>0.90$ & $29(9.1)$ & $29(23.4)$ & $58(13.1)$ & $<0.001 * *$ \\
\hline \multicolumn{5}{|c|}{ Waist (cm) } \\
\hline$<80.0$ & $163(51.1)$ & $39(31.5)$ & $202(45.6)$ & $<0.001 * *$ \\
\hline $80-85$ & $69(21.6)$ & $26(21)$ & $95(21.4)$ & 0.946 \\
\hline $85-88$ & $25(7.8)$ & $16(12.9)$ & $4 I(9.3)$ & 0.192 \\
\hline$>88$ & $62(19.4)$ & $43(34.7)$ & $105(23.7)$ & $0.00 I^{* *}$ \\
\hline Total & $319(100.0)$ & $124(100.0)$ & $443(100.0)$ & - \\
\hline
\end{tabular}

Results are presented as No (\%)

$P$ values are obtained using the Chi-square test/ $2 \times 2$ Fisher Exact test

*Moderately significant $0.01<P \leq 0.05$

**Strongly significant $\mathrm{P} \leq 0.01$

Subjects in the younger age group had significantly $(\mathrm{p}=<0.001)$ lower WHR while, women aged $>30 \mathrm{y}$ were found to have higher WHR $(>0.80)$.

Recently, waist circumference has been found to be an even better marker of central obesity than WHR. Accordingly, the National Heart,
Lung, and Blood Institute Clinical Guidelines recommend the use of waist circumference in addition to BMI in clinical screening of adults. For Asian Indians, the optimum waist circumference is $<80 \mathrm{~cm}$ in women and $<90 \mathrm{~cm}$ in men. These values are about 8 to $10 \mathrm{~cm}$ lower than that recommended for Whites and underscore the need for early institution of weight management programme. Accordingly, it 
was observed that $60 \%$ of the subjects had $\mathrm{WC}>80 \mathrm{~cm}$. Younger age group subjects had significantly $(\mathrm{p}=<0.001)$ lower $\mathrm{WC}(<80 \mathrm{~cm})$ while Women $>30 \mathrm{y}$ were found to have higher $\mathrm{WC}>80 \mathrm{~cm}$. Classification according to menopausal status indicated that Pre M subjects had lower WHR \& WC while, Post menopausal women had higher than desirable values.

\section{Life style practices}

Majority $(60 \%)$ of the subjects followed a 3 meal pattern. $67 \%$ were non vegetarians (Table 9). Consumption of Non vegetarian food item was significantly prevalent among the Pre $\mathrm{M}$ group of women in all the professional groups, with a significant number $(p<0.001)$ from health care sector. It can be presumed that religion and work schedule also dominated the choice of non vegetarian foods. General diet habits in respect to work environment showed that consumption of health drinks by Pre $\mathrm{M}$ subjects was found to be moderately high $(\mathrm{P}<0.030)$ among teachers and low in healthcare professionals. A similar pattern was observed in Post M women.

Table 9 General Food habits of the study

\begin{tabular}{|c|c|c|c|c|c|c|c|c|c|c|}
\hline \multirow{2}{*}{$\begin{array}{l}\text { Diet } \\
\text { pattern }\end{array}$} & \multicolumn{2}{|c|}{ Teachers } & \multicolumn{2}{|c|}{ Health care } & \multicolumn{2}{|l|}{ Clerical } & \multicolumn{2}{|l|}{ Total } & \multirow{2}{*}{$\begin{array}{l}\text { P value } \\
\text { (Pre) }\end{array}$} & \multirow{2}{*}{$\begin{array}{l}\text { P value } \\
\text { (Post) }\end{array}$} \\
\hline & $\begin{array}{l}\text { Pre M } \\
(n=\mid 30)\end{array}$ & $\begin{array}{l}\text { Post M } \\
(n=5 I)\end{array}$ & $\begin{array}{l}\text { Pre M } \\
(n=\mid 0 I)\end{array}$ & $\begin{array}{l}\text { Post M } \\
(n=21)\end{array}$ & $\begin{array}{l}\text { Pre M } \\
(n=88)\end{array}$ & $\begin{array}{l}\text { Post M } \\
(n=52)\end{array}$ & $\begin{array}{l}\text { Pre M } \\
(n=3 \mid 9)\end{array}$ & $\begin{array}{l}\text { Post M } \\
(n=\mid 24)\end{array}$ & & \\
\hline \multicolumn{11}{|l|}{ Diet type } \\
\hline Vegetarian & $69(53.0)$ & $26(50.9)$ & $29(28.7)$ & $6(28.5)$ & $64(72.7)$ & $29(55.7)$ & $161(50.4)$ & $6 I(49.1)$ & $<0.00 I^{* *}$ & 0.1 \\
\hline Mixed & $6 I(46.9)$ & $25(49.0)$ & $72(71.2)$ & $15(71.4)$ & $24(27.3)$ & $23(44.2)$ & $158(49.5)$ & $63(50.8)$ & $<0.00 I^{* *}$ & 0.1 \\
\hline \multicolumn{11}{|l|}{ Snacks } \\
\hline Never & $40(30.7)$ & $2 I(4 I . I)$ & $26(25.7)$ & $4(19.0)$ & $3 I(35.2)$ & $15(28.8)$ & $97(30.4)$ & $40(32.2)$ & 0.37 & 0.1 \\
\hline I - 2/wk & $2(1.5)$ & $4(7.8)$ & $20(19.8)$ & $4(19.0)$ & $56(63.6)$ & $37(71.1)$ & $78(24.4)$ & $45(36.3)$ & $<0.00 I^{* *}$ & $<0.00 I^{* *}$ \\
\hline Daily & $88(67.6)$ & $26(50.9)$ & $55(54.4)$ & $13(61.9)$ & $\mathrm{I}(\mathrm{I} . \mathrm{I})$ & - & $144(45.1)$ & $39(31.4)$ & $<0.00 I^{* *}$ & $<0.00 I^{* *}$ \\
\hline \multicolumn{11}{|l|}{ Coffee } \\
\hline Never & $43(33.1)$ & $23(45.1)$ & $33(32.6)$ & $5(23.8)$ & $30(34.1)$ & $20(38.5)$ & $106(33.2)$ & $48(38.7)$ & 0.98 & 0.2 \\
\hline $2-3 /$ day & $\mathrm{I}(0.7)$ & 0 & $2(1.9)$ & $\mathrm{I}(4.7)$ & $50(56.8)$ & $27(51.9)$ & $53(16.6)$ & $28(22.5)$ & $<0.00 I^{* *}$ & $<0.00 I^{* * *}$ \\
\hline$>3 /$ day & $86(66.1)$ & $28(54.9)$ & $66(65.3)$ & I5(7|.4) & $8(9.1)$ & $5(9.6)$ & $160(50.1)$ & $48(38.7)$ & $<0.001 * *$ & $<0.00 I^{* *}$ \\
\hline \multicolumn{11}{|l|}{ Tea } \\
\hline Never & $53(40.7)$ & $24(47.1)$ & $29(28.7)$ & $4(19.0)$ & $18(20.4)$ & $16(30.7)$ & $100(31.3)$ & $44(35.4)$ & $0.005 * *$ & $0.05 I^{*}$ \\
\hline $2-3 /$ day & $\mathrm{I}(0.7)$ & 0 & $2(1.98)$ & 0 & $59(67.0)$ & $3 I(59.6)$ & $62(19.4)$ & $3 I(25.0)$ & $<0.001 * *$ & $<0.00 I^{* *}$ \\
\hline$>3 /$ day & $76(58.4)$ & $27(52.9)$ & $70(69.3)$ & I7(80.9) & $\mathrm{II}(12.5)$ & $5(9.6)$ & 157(49.2) & $49((39.5)$ & $<0.001 * *$ & $<0.00 I^{* * *}$ \\
\hline \multicolumn{11}{|l|}{ Fruits } \\
\hline Never & $6(4.6)$ & $3(5.8)$ & $4(3.9)$ & 0 & $3(3.4)$ & $5(9.6)$ & $13(4.1)$ & $8(6.5)$ & 0.91 & 0.3 \\
\hline I - 2/wk & $25(19.2)$ & $9(17.6)$ & $22(21.7)$ & $5(23.8)$ & $85(96.6)$ & $47(90.38)$ & $132(4 \mid .3)$ & $61(49.1)$ & $<0.00 I^{* *}$ & $<0.00 I^{* *}$ \\
\hline$>2 / w k$ & $99(76.1)$ & $39(76.4)$ & $75(74.2)$ & $16(76.2)$ & - & - & $174(54.5)$ & $55(44.3)$ & $<0.00 I^{* *}$ & $<0.00 I^{* *}$ \\
\hline \multicolumn{11}{|c|}{ Health drinks } \\
\hline No & $80(61.5)$ & $37(72.5)$ & $78(77.2)$ & $17(80.9)$ & $56(63.6)$ & $39(75.0)$ & $215(67.3)$ & $93(75.0)$ & $0.030 *$ & 0.8 \\
\hline Yes & $50(38.4)$ & $14(27.4)$ & $23(22.7)$ & $4(19.0)$ & $32(36.3)$ & $13(25.0)$ & $104(32.6)$ & $3 I(25.0)$ & $0.030 *$ & 0.8 \\
\hline
\end{tabular}

Results are presented in No (\%)

$P$ values are obtained using the chi-square test $/ 2 \times 3$ fisher Exact test

*Moderately significant $0.01<P \leq 0.05$

**Highly significant $\mathrm{P} \leq 0.01$

+Suggestive significance

Every day snacking during break time was practiced by majority of $\quad M$ women belonging to teaching and health care group. A minimum of the subjects, it was observed to be significantly high, in Pre $M$ and Post

2-3 cups of coffee/ tea per day was being consumed by the subjects. 
Consumption of $>3$ cups of coffee/tea per day was observed to be higher in the Pre M group. The Pre M and Post M women belonging to teaching and health care category were observed to consume these beverages frequently. Fruits a high source of antioxidants with anti atherogenic properties was found to be frequently consumed only by teachers and health care professionals. It was of interest to note that a significant number of women from Clerical sector either consumed it occasionally or never consumed. This can be attributed to the high market prices.

\section{Medical history}

Majority $(60 \%)$ of the Pre $M$ women never underwent annual medical check up, while $62 \%$ of the Post $\mathrm{M}$ women reported one general medical check up in the past 2years. However, blood tests and procedures for preventive cardiac care were not investigated. An overall of $37 \%$ Post $\mathrm{M}$ women were on treatment for various conditions like hypertension, diabetes, arthritis and allergy. 70\% of post M women were found to have Blood pressure $>120 / 80 \mathrm{~mm}$ $\mathrm{Hg}$. Post $\mathrm{M}$ women from teaching professional were found to have significantly higher Blood pressure $(\mathrm{p}=<0.001)$.

Presence of CVD risk factors as reported by the subjects is presented in Table 10. Women aged $>30$ years were observed to have higher incidence of CVD risk factors as compared to younger subjects. Incidentally, all the CVD risk factors were higher in the Post $\mathrm{M}$ women than Pre M women. However occurrence of Hypothyroidism was found to be high among the Pre M group of women.

Table 10 Cardiovascular disease risk factorst in Pre $M$ and Post $M$ subjects

\begin{tabular}{|c|c|c|c|c|c|}
\hline \multirow[t]{2}{*}{ Family History } & \multicolumn{2}{|c|}{ Pre-Menopause $(n=319)$} & \multicolumn{2}{|c|}{ Post-Menopause $(n=\mid 24)$} & \multirow[t]{2}{*}{ P value } \\
\hline & No & $\%$ & No & $\%$ & \\
\hline \multicolumn{6}{|l|}{ Hypertension } \\
\hline Absent & 293 & 91.8 & 90 & 72.6 & $<0.001 * *$ \\
\hline Present & 26 & 8.2 & 34 & 27.4 & \\
\hline \multicolumn{6}{|l|}{ DM } \\
\hline Absent & 311 & 97.5 & 98 & 79.1 & $<0.00 I^{*} * *$ \\
\hline Present & 8 & 2.5 & 26 & 20.9 & \\
\hline \multicolumn{6}{|l|}{ Hypothyroidism } \\
\hline Absent & 305 & 95.6 & 122 & 98.4 & 0.255 \\
\hline Present & 14 & 4.4 & 2 & 1.6 & \\
\hline
\end{tabular}

$P$ values are obtained using the Chi-square test/ $2 \times 2$ Fisher Exact test

†Self reported

*Moderately significant $0.01<p \leq 0.05$

$* *$ Strongly significant $p \leq 0.0$ I

\section{Family history for CVD risk factors}

Hereditary factor is one of the main non modifiable risk factor for the occurrence of cardio vascular diseases. Prevalence (self reported) of CVD risk factors among the family members of the study group. Figure 2 showed that incidence of Dyslipidemia was high among the female members of the family (sisters and mothers). Diabetes was found to be prevalent in both parents in comparison to grandparents. Within the family, prevalence of diabetes was found to be higher among the 'Mothers' and 'Brothers' when compared to 'Fathers' and 'sisters'. Similarly incidence of Hypertension was found to be high among mother and brother. However, CHD was comparatively high among the male members of the family (Brothers and Fathers).

In general, it was of interest to observe that the mother's in the family showed higher prevalence for dyslipidemia, diabetes and hypertension. Among the siblings, brothers showed higher prevalence for diabetes, hypertension and coronary heart disease while, sisters exhibited higher prevalence for dyslipidemia.

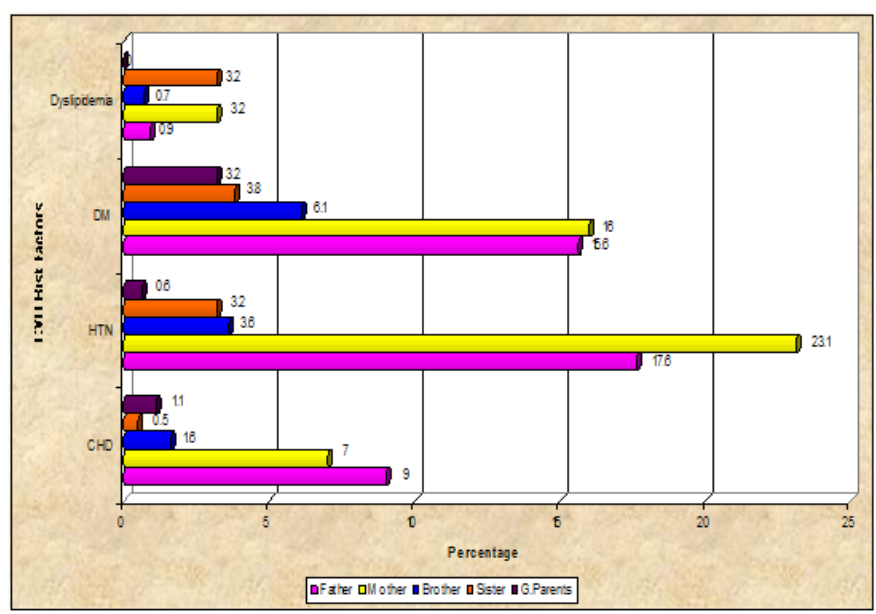

Figure 2 CVD risk factors. 


\section{Discussion}

Demographic shifts and developmental transition has escalated obesity and other chronic non communicable diseases. The major factors implicated in this phenomenon are occupational patterns, dietary pattern and lifestyle changes. Increase in literacy and socioeconomic status, in addition to lifestyle factors particularly dietary habits and physical activity appear to be important determinants in predisposing a population to develop cardiovascular risk factors and other chronic diseases.

The objective of promoting employment for the women is to improve the quality of life by increasing the total household income, ${ }^{11}$ the average income of the study group ( $>$ Rs 8000/) indicated a satisfactory economic status. It is assumed that gainful employment of women outside home would lead to higher levels of net income which in turn will provide higher living standard, including food consumption and improved nutritional status of the family. However, these assumptions are controversial in relation to the gainful employment of women outside home while some studies have reported a positive link ${ }^{12}$ between increased income and nutrition, others have reported a decline in nutritional status of women. ${ }^{13,14}$

The onset of natural menopause occurs around 45-52years in Indian population. while, Caucasian women report a median of 51.4years, Japanese 50.4years, Malaysians 50.7years, Chinese 47years and Thai women 49.5years (lower socioeconomic group of women). ${ }^{15}$ In the present study majority of the post menopausal women had attained natural menopause between the ages 40-50years with a median age of 46years. The age at menopause appears to be different in various ethnic groups which may be attributed to food habits and other physiological influences.

By and large the educational levels of these women were better when compared to the reported figures for women in India. ${ }^{16,17}$ The women's acceptability of family welfare measures (tubectomy) with profession revealed to be highest in clerical group of subjects. Though 'education' was found to influence the demographic behaviour in terms of family size and family planning, it did not appear to promote appropriate food behaviour, a trend towards preference for foods rich in salt, sugar and fat in terms of "every day" snacking during break time, while at work was observed among all the subjects. Hence, the absence of awareness of their nutritional needs despite a satisfactory educational level and improved standard of living may not promote good nutrition on the other hand may adversely affect energy excesses and micronutrient deficiencies particularly, that of iron and vitamins having antioxidant properties which are essential in slowing down the process of ageing and development of associated disorders and chronic diseases.

In the recent past, awareness and utilization of health services has increased particularly among urban Indians, due to privately funded health care services, corporate type hospitals and introduction of several health insurance schemes by central and state governments. In general all the subjects in both categories did not view menopause as linked to disease or medical care. Majority of the subjects had undergone medical check-up pertaining to gynaecological/ obstetrics problems. Most of the Pre menopausal subjects did not feel it necessary to undergo cardiac evaluation while, many post menopausal subjects expressed underlying fear of being diagnosed for any chronic diseases including CVD. Although women in the healthcare sector had better access to medical facilities than the teachers and clerical category of subjects importance was not attached to periodic check up for risk profiles.

Irrespective of the profession or menopausal status majority of the women appeared to be 'heavier' (Pre M- 59.6kg, Post M$63.0 \mathrm{~kg}$ ) and taller (Pre M $156.7 \mathrm{~cm}$, Post M $155.1 \mathrm{~cm}$ ) as compared to NNMB reported values for urban Indian women - weight $52.2 \mathrm{~kg}$, height $-154.9 \mathrm{~cm}$. However, post menopausal women were shorter than pre menopausal women. Short stature may be an adaptation to protein energy malnutrition and or specific nutrient deficiencies during childhood ${ }^{18}$ or kyphoscoliosis, which usually occurs during menopausal phase due to osteoporosis related to insufficiency of estrogen. ${ }^{19}$

Body size in adults, widely used as an indicator of overall health and nutritional status also serves as pointers to the socio economic status in the population. Although, increasing trend in weight, BMI, WC, WHR, MUAC, TSF and MUAMC against age gradient is described as age related phenomena, greater BMI, WC, WHR and low MUAC, MUAMC among the subjects ( $>30 \mathrm{yrs})$ as compared to standards ${ }^{20,21}$ for Indian women are indicative of the fact that these factors are likely to contribute to the aging process which in turn can influence the development of chronic disease.

Asian Indians have greater abdominal fat and visceral fat even at normal BMI and are more susceptible to obesity co-morbidities and syndrome X, a contributing factor for risk of CVD. ${ }^{22,23}$ Urban Indians have body composition conducive to development of dyslipidemia. ${ }^{24}$ In the present study, younger women (20-29yrs) with low BMI, WC and WHR exhibited high TSF values that could predispose them to dyslipidemia and obesity with increase in age.

Obesity is also a well established risk factor for chronic diseases and other cardio vascular risk factors. The most widely used indices to measure obesity are BMI. The World Health organization recommends a BMI of $<18.5$ as indicative of chronic energy deficiency, 18.5-22.9 as normal, 23-24.9 as overweight or at risk and $>25$ as obese for Asian Indians. ${ }^{20}$ Accordingly, the subjects were either overweight (20\%) or obese (42\%) irrespective of profession. Post menopausal women (Post M) had significantly $(\mathrm{p}=0.010)$ higher mean BMI (26.3) as compared to Pre menopausal women (Pre M). BMI showed increasing trend with age, younger subjects $(<29 \mathrm{y})$ had $\mathrm{BMI}<21.7$ while, older women ( $\geq 30$ years) had $\mathrm{BMI} \geq 24.0$. A study on a general population of South Indian women, ${ }^{25}$ indicates that the optimal cut off values to predict hypertension is $\geq 24.8$ and diabetes $\geq 24.2$. In this context the subjects aged 30years and above, irrespective of the menopausal status appear to be at risk for development of both hypertension and diabetes.

The prevalence of obesity is increasing in countries with economic transition however; it is highly variable between developing countries and within population. General prevalence of obesity as measured by BMI is higher in women than in men. Studies by Nutrition Foundation of India have documented that prevalence of obesity in women is double that of men among Delhi urban employees.,26 Similarly the subjects aged $30 \mathrm{yrs}$ and above, irrespective of the profession, belonging to an urban population of Mysore city, also exhibited higher than the desirable values for BMI, recommended for Indians. No comparisons could be made with male gender as it was not within the scope of the study.

The general measure of central obesity is WHR. WHR $>0.80$ corresponds with central obesity, a pattern of android fat distribution 
that increases risk for CVD and its risk factors. ${ }^{27,28}$ Menopause a nonmodifiable risk factor predisposes women to android obesity and the increase is greater when compared to pre menopausal women. ${ }^{29}$ Adverse metabolic consequences are seen with central or android obesity. The fat in android obesity is more sensitive to catecholamines and less sensitive to insulin with resulting hyper-insulinemia causing more chances of diabetes mellitus and coronary heart disease (CHD) and atherogenic lipid profile, hypertension, atherosclerosis. ${ }^{30}$ This risk appears to be more in Asian Indians. WHR of the subjects aged $>30 y$ rs showed an increasing trend with age, there is likelihood of these subjects being exposed to altered cardio metabolic risk if suitable preventive strategies are not adapted.

Recently waist circumference (WC) has been found to be a better marker for central obesity than WHR..$^{31,32}$ For Asian Indians the optimum waist circumference is $<80 \mathrm{~cm}$, these values are $8-10 \mathrm{~cm}$ lower than that recommended for whites. The subjects were evaluated for $\mathrm{WC}$ and it showed an increasing trend with age. Abdominal obesity is associated with elevated levels of several important risk factors for atherosclerotic disease, such as hypertension, and increased lipid, glucose and insulin levels. Selected subjects were evaluated for few of these parameters in the $2^{\text {nd }}$ phase of the study.

Evaluation of the health and family history in an individual is an important aspect of risk assessment associated with CVD. A family history of diabetes and hypertension were the mostly frequently reported health problems among the family members of both pre and post-menopausal women. A higher frequency was reported among the parents and mother in particular of the pre-menopausal subjects. Family history was a strong independent risk factor, with $70 \%$ of the subjects having either or both parents with diabetes, hypertension and cardiac event or with a combination of these risk factors. The possibility that these events are related at menopause or during the transitional phase, in Indian women belonging to general population has not been extensively investigated. Hence, comparisons of relevant parameters, was not possible in this phase.

\section{Conclusion}

The subjects above the age of 30years irrespective of their profession had higher BMI, WHR, WC, TSF and low protein status, a predisposition factor for CVD. The dietary preferences for snacking and higher intake of coffee and tea that increased the consumption of sugar and salt per day, low physical activity and family history for cardiovascular events and risk factors can contribute to acceleration of altered cardio bio markers and thereby promote adverse health effects. Lack of motivation and awareness to undergo annual cardiac evaluation, places them at a higher risk for cardiac events in the future. The study suggests that hereditary factors may be super imposed by environmental factors for development of risk factors for cardiovascular disease, and needs early detection in the form of annual medical check - up and self-assessment of body measures for all women above $30 \mathrm{yrs}$ of age. Although, the postmenopausal women exhibited a higher obesity status, it was surprising to note that the premenopausal women displayed similar features, thus, placing them at equal risk for CVD during the menopausal transition.

\section{Acknowledgements}

The authors acknowledge the participation of subjects and the respective Institutes/ organizations for permitting to carry out the study.

\section{Conflict of interest}

The author declares no conflict of interest.

\section{References}

1. Mohan V, Rao GHR. Type 2 Diabetes in South Asians: Epidemiology, Risk Factors and Prevention. 1st ed. India: Jaypee Brothers Medical Publishers; 2007.

2. Prasad DS, Kabir Z, Dash AK, et al. Abdominal obesity, an independent cardiovascular risk factor in Indian subcontinent: A clinico epidemiological evidence summary. J Cardiovasc Dis Res. 2011;2(4):199-205.

3. Marie Ng, Tom Fleming, Margaret Robinson, et al. Global, regional, and national prevalence of overweight and obesity in children and adults during 1980-2013:a systematic analysis for the Global Burden of Disease Study 2013. Lancet. 2014;384(9945):766-781.

4. Zhou B. Diet and cardiovascular disease in China. In: Shetty P, Gopalan $\mathrm{C}$, editors. Diet, nutrition and chronic disease: an asian perspective. UK: Smith-Gordan and Co Ltd; 1998.

5. Murray CJL, Lopez AD. Global comparative assessments in the health sector. USA: World health Organization, National Academies Press; 1994.

6. Yusuf S, Reddy S, Ounpuu S, et al. Global burden of cardiovascular diseases: part 1:general considerations, the epidemiologic transition, risk factors, and impact of urbanization. Circulation. 2001;104(22):2746 2753 .

7. Nanette Santoro, Steven R Goldstein. Textbook of Peri-menopausal Gynecology. Taylor and Francis Group, USA: CRC Press; 2002.

8. Kritz Silverestein D, Barrett Corner E. Long term postmenopausal hormone use, obesity and fat distribution in older women. JAMA. 1996;275(1):46-49.

9. Jelliffee DB. The assessment of the nutritional status of the community, (with special reference to field surveys in developing regions of the world). Monogr Ser World Health Organ. 1966;53:3-271.

10. Rosalind S Gibson. Principles of nutritional assessment. 2nd ed. UK: Oxford University Press; 2005. 291 p.

11. Papola, Trilok S. Employment of women in south Asian Countries. Ind J of Labor Econ. 1993;36(1):48-56.

12. Office of the Registrar General (1992, 1993, 1994), Office of the Registrar General and census commissioner (1987,1992, 2001) Ministry of Family welfare 1991, 1992, 2001.

13. Srikantia, Soumya $G$ Rao. Nutritional deficiency diseases. In: Gopalan C, Kaur S, editors. Women and nutrition in India. Nutrition Foundation of India, Special publication series; 1989. 5:224.

14. Anuurad E, Shiwaku K, Nogi A, et al. The new BMI criteria for Asians by the regional office of the western Pacific region of WHO are suitable for screening of overweight to prevent metabolic syndrome in elder Japanese workers. J Occup Health. 2003;45(6):335-343.

15. Kuppuswamy V, Gupta S. oronary heart disease in south Asians. Practitioner. 2003;247(1644):181-190.

16. Misra A, Kalpana Luthra, Vikram NK. Dyslipidemia in Asian Indians: Determinants and Significance. J Assoc Physicians India. 2004;52:137142 .

17. WHO Expert Consultation. Appropriate body mass index for Asian populations and its implications for policy and intervention strategies. Lancet. 2004;363(9403):157-163. 
18. Nelas L, Christiansen C. The Pathophysiology of peri and post menopausal bone loss. Br J Obes and Gyne. 1989;96(5):580-587.

19. Mark LW, Jonathan M Hodgson, Frank M Ng, et al. The role of nutrition in abdominal obesity. Nutrition research. 1999;19(1):85-101.

20. WHO Regional Report. The Asia-Pacific perspective redefining obesity and its treatment. Australia: Health Communications Australia Pvt Limited; 2000

21. Rosemary Kevany, Mahshid Lotfi, John Mason. women and nutritionnutrition policy discussion paper. 1990:6.

22. Sybil L Crawford, Catherine B Johannes. The epidemiology of cardiovascular disease in post menopausal women. Journal of Clinical endocrinology \& metabolism. 1999;84(6):1803-1812.

23. Hjortland MC, McNamara PM, Kannel WB. Some atherogenic concomitants of menopause: the Framingham Study. Am J Epidemiol. 1976;103(3):304-311.

24. Kuller LH, Gutai JP, Meilahn EN, et al. Relationship of endogenous sex steroid hormones to lipids and apo-proteins in postmenopausal women. Atherosclerosis. 1990;10(6):1058-1066.

25. Manson JE, Colditz GA, Stampfer MJ, et al. A prospective study of obesity and risk of coronary heart disease in women. $N$ Engl J Med. 1990;322(13):822-889.
26. Gopinath N, Chadha SI, Jain P, et al. Epidemiological study of obesity in adults in urban populations of Delhi. J Assoc Physicians India. 1994;42(3):212-215.

27. Enas A Enas, Senthilkumar A Coronary artery disease risk in asia indians: an update and review. In: Gundu Rao HR, Thanikachalam S, editors. Coronary Artery Disease: risk promoters, pathophysiology and prevention. south Asian society on atherosclerosis and thrombosis. India: JAPEE Medical publisher; 2005. p. 21-57.

28. Rexrode KM, Carey VJ, Hennekens $\mathrm{CH}$, et al. Abdominal adiposity and coronary heart disease in women. JAMA. 1998;280(21):1843-1848.

29. Christopher J Ley, Belinda Lees, John C Stevenson. Sex and menopause-associated changes in body-fat distribution. Am J Clin Nutr. 1992;55(5):950-954.

30. Arup Kumar Majhi. Obesity and Women's Health. J Obs \& Gynae VIII. $2003 ;(5): 245-249$.

31. Enas EA, Senthilkumar A, Juturu V, et al. Coronary artery Disease in women. Indian Heart J. 2001;53(3):282-292.

32. Ford S Earl. Prevalence of the metabolic syndrome defined by the international diabetes federation among Adults in the us. Diabetes Care. $2005 ; 28(11): 2745-2749$. 